\title{
ON AN EXTENSION OF THE VON KÁRMÁN-TSIEN METHOD TO TWO-DIMENSIONAL SUBSONIC FLOWS WITH CIRCULATION AROUND CLOSED PROFILES*
}

\author{
BY \\ C. C. LIN \\ Brown University
}

1. Introduction. The method for treating compressible flows, as developed by Chaplygin, ${ }^{1}$ von Kármán ${ }^{2}$ and Tsien ${ }^{3}$ leads to a successful solution of the flow pattern past solid bodies when the flow has no circulation. When the flow has a finite circulation, as in the case of airfoils, the profile shapes furnished by this theory are not closed. It is doubtless desirable to develop the theory so as to remove this difficulty.

Recently, Bers ${ }^{4}$ succeeded in obtaining flows with circulation around closed profiles. As is usual in the case of a first success, the new method has a few disadvantages. In the first place, the mapping between the actual compressible flow and the associated incompressible flow is not regular at the stagnation points. Thus, if the profile in the associated incompressible flow is regular everywhere, angular points would appear in the profile in the compressible case; and vice versa. The application of the method is further complicated by the fact that the angle thus generated depends on the free-stream Mach number. For the engineer, the treatment has the additional inconvenience of involving the concepts of Riemannian geometry (which are avoided in the present treatment).

In the present article we shall describe a method which is free from the disadvantages mentioned above. The derivation is very simple, and no reference is made to Riemannian geometry. Yet the result includes all the previous ones as special cases. Indeed, the treatment seems to be now in the most natural and the most general form which is obtainable from the line of study of Chaplygin, von Kármán and Tsien. It also has great flexibility. Given one incompressible flow, there is still an analytic function at our disposal for constructing compressible flows. This freedom of choice enables us to avoid much unnecessary numerical labor in constructing flows of certain general types. A large number of compressible flows can be derived from a given incompressible flow by the present method without numerical integration.

Apart from giving a useful method for constructing compressible flow patterns, the present development has the following significance. First, the freedom of disposing of one analytic function leads to the solution of the direct problem,- - namely to

* Received May 18, 1946.

1 S. A. Chaplygin, On gas jets. Scientific Memoirs, Moscow Univ., Math. Phys. Sec. 21, 1-121 (1902). (English translation published by Brown University, 1944. Also NACA TM No. 1063, 1944.)

2 Th. von Kármán, Compressibility effects in aerodynamics. Jour. Aero. Sci. 8, 337-356 (1941).

3 Hsue-Shen Tsien, Two-dimensional subsonic flow of compressible fluids. Jour. Aero. Sci. 6, 339-407 (1939).

4 L. Bers, On a method of constructing two-dimensional subsonic compressible flows around closed profiles, NACA TN No. 969 (1945); On the circulatory subsonic flow of a compressible fluid past a circular cylinder, NACA TN No. 970 (1945). See also S. Bergman, On two-dimensional flows of compressible fluids, NACA TN No. 972 (1945). 
calculate the compressible flow past a given profilc. Indeed, the solution of problems of compressible flow, either direct or inverse,-- namely, the construction of flows around profiles either given beforchand or not-is now an a parallel footing with the incompressible case. In either case, the direct problem requires a method of successive approximations. ${ }^{5}$ Secondly, the application of the pressure coefficient formula of von Kármán and Tsien to flows with circulation is justified on the same basis as in the circulationfree case. Experimentally, the formula has been found to be successful even when there is circulation, although the theory has so far been incomplete. The original development of von Kármán and Tsien leaves the body not closed, while the profiles given by the method of Bers do not have the same analytic nature in the incompressible and the compressible cases. The present method removes these difficulties.

Further investigations of the significance of the present method are being carried out. The present article contains only the essential result and its proof. It is hoped that a complete discussion of further developments may be published very soon.

2. Method of constructing two-dimensional subsonic flows with circulation around profiles. Let $p, \rho, u, v$ be the pressure, density and components of velocity of a steady two-dimensional irrotational flow in the $x, y$ plane. Let $p$ be a function of the density $\rho$ only (given either by the isentropic relation or any other approximate relation). Then there exist the velocity potential $\phi$ and the stream function $\psi$ defined by the following differential relations:

$$
\begin{aligned}
& d \phi=u d x+v d y, \\
& d \psi=-\rho v d x+\rho u d y .
\end{aligned}
$$

The velocity components $u, v$ and the density $\rho$ are further connected by Bernoulli's equation

$$
\frac{q^{2}}{2}+\int \frac{d p}{\rho}=\text { const., } \quad\left(q^{2}=u^{2}+v^{2}\right) .
$$

It is convenient to refer the density of the gas to that at the stagnation point and to refer all the velocities to the velocity of sound at stagnation. The coordinates $x, y$ may be regarded as referred to the size of the body, and the pressure as referred to the product of stagnation density and the square of the velocity of sound at stagnation. Throughout this article, this process shall be implied, and all the quantities under discussion are dimensionless.

As is well-known, the problem simplifies greatly if the pressure-density relation is approximated by

$$
p=A-\frac{B}{\rho} .
$$

This is the basis of the method of Chaplygin, von Kármán and Tsien. A discussion of its physical interpretation may be found in the papers of these authors. Equation (2.3) leads to

For the incompressible case, see T. Theodorsen, Theory of wing sections of arbitrary shapes, NACA Rep. No. 411 (1931).

T. Theodorsen and I. E. Garrick, General potential theory of arbitrary wing sections, NACA Rep. No. 452 (1933). 


$$
c=\frac{1}{\rho}=\sqrt{1+\overline{q^{2}}}
$$

where $c$ is the local velocity of sound. Indeed, $B$ must be equal to unity if the reference stagnation quantities are calculated from (2.3) by the use of (2.4)

Under the approximation (2.4), the following method may be used for constructing two-dimensional subsonic flows with circulation around closed profiles.

Given an incompressible flow past a profile $P_{0}$ in the $\zeta$-plane $(\zeta=\xi+i \eta)$ described by the complex stream function $F(\zeta)$ and the complex velocity $w_{0}(\zeta)$, choose a function $k(\zeta)$, regular in the region exterior to $P_{0}$ and including the point at infinity, having no root in $R_{0}$, and such that $R_{0}$

and that

$$
\left|\frac{1}{2} w_{0}(\zeta)\right|<|k(\zeta)|<\infty \quad \text { on } \quad P_{0}
$$

$$
\oint k(\zeta) d \zeta-\frac{1}{4} \oint \frac{\overline{w_{0}^{2}(\zeta)}}{k(\zeta)} d \zeta=0
$$

where the integration is performed along any contour enclosing $P_{0}$. Then

$$
\begin{aligned}
x+i y & =\int k(\zeta) d \zeta-\frac{1}{4} \int \overline{\frac{w_{0}^{2}(\zeta)}{k(\zeta)} d \zeta,} \\
\frac{2 q}{1+\sqrt{1+q^{2}}} e^{-i 0} & =\frac{w_{0}(\zeta)}{k(\zeta)}, \\
\phi+i \psi & =F(\zeta)
\end{aligned}
$$

gives the parametric representation of a compressible flow past a profile $P$ in the $x, y$ plane with $\zeta$ as parameter, where $P$ has the same general analytic nature (e.g. same number of corners, etc.) as the original profile $P_{0}$. In these formulae, $\phi, \psi$ are the velocity potential and stream function defined by (2.1) and $q, \theta$ denote the magnitude and direction of the velocity of the compressible flow.

3. Proof. The proof consists of two parts. First, it is necessary to show that, after the auxiliary variable $\zeta$ is eliminated from (2.8)-(2.10), we obtain proper functional relations between $\phi, \psi, q, \theta$ and $x, y$. Secondly, we must show that the profile $P$ is a closed curve and is mapped into $P_{0}$ by a regular mapping such that the regions $R$, exterior to $P$, and $R_{0}$, exterior to $P_{0}$, are mapped into each other in a one-to-one manner.

(a) The first part of the proof is simple. It is well known ${ }^{6}$ that under the approximation (2.4), the relations

with $z, F, w^{*}$ defined by

$$
d z=\frac{d F}{w^{*}}-\frac{1}{4} \overline{w^{*} d F}, \quad F=F\left(w^{*}\right)
$$

$$
\left.\begin{array}{rl}
z & =x+i y, \\
F & =\phi+i \psi, \\
w^{*} & =\frac{2 q}{1+\sqrt{1+q^{2}}} e^{-i \theta}
\end{array}\right\}
$$

- Cf., for example, Eqs. (23), (26) of Tsien's paper quoted in Footnote 3. 
would give a solution of the differential equations of compressible flow. Instead of trying to establish a relation between $F$ and $w^{*}$ directly, we introduce an auxiliary variable $\zeta$ such that $F(\zeta)$ and $w^{*}(\zeta)$ are analytic functoins. It is well known that great simplification is obtained by taking $F(\zeta)$ to be the complex potential of an incompressible flow similar to the compressible flow we desire. However, the extent of arbitariness in the choice of $w^{*}(\zeta)$ has not been carefully examined. It is clear that any choice of $w^{*}(\zeta)$ will be sufficient so far as satisfying the differential equations is concerned.

In the present case we dispose of the arbitrary function by writing

$$
w^{*}(\zeta)=w_{0}(\zeta)_{i}^{\prime} k(\zeta)
$$

The only requirements on $k(\zeta)$ are the general conditions of regularity and the relations (2.6) and (2.7), which will be discussed immediately.

(b) The second part of the proof is also very simple. In the first place, the profile $P$ is closed by virtue of (2.7). ${ }^{6}$ The regularity of the mapping is established if the Jacobian of the transformation maintains the same sign and does not vanish or become infinite in the region $R_{0}$, including the boundary $P_{0}$ and the point at infinity. It can be easily verified that the Jacobian is

$$
J=\frac{\partial(x, y)}{\partial(\xi, \eta)}=\operatorname{Im}\left\{\bar{z}_{\xi z_{\eta}}\right\}=|k|^{2}\left\{1-\frac{1}{16}\left|\frac{w_{0}}{k}\right|^{4}\right\} .
$$

From this expression, it is clear that the requirement is satisfied when $k$ satisfies the restrictions specified in the last section.*

4. Discussion. (a) The function $k(\zeta)$. To make use of the freedom in choosing the function $k(\zeta)$ is the essential improvement made in the present paper. Von Kármán and Tsien gave an interpretation of $w^{*}(\zeta)$ by identifying it with the complex velocity $w_{0}(\zeta)$ in the associated incompressible flow. This means that they put

$$
k(\zeta) \equiv 1 .
$$

They were therefore unable to meet the requirement (2.7) for closing the profile, for flows with circulation. Bers overcame this difficulty by virtually taking

$$
k(\zeta)=\text { const. }\left\{w_{0}(\zeta)\right\}^{1-\sqrt{1-M_{\infty}^{2}}},
$$

where $M_{\infty}$ is the free-stream Mach number. However, at the stagnation points of the incompressible flow

$$
|k(\zeta)|=0 \text {, }
$$

and the mapping between the profiles $P$ and $P_{0}$ is not regular there. In applying his method to the calculation of compressible flow past a circle, Bers had to start with the rather complicated problem of finding the incompressible flow past two intersecting circular arcs with the stagnation points at the points of intersection and with the

* Note added in proof: Professor K. O. Friedricks pointed out to the author that, in the compressible as well as the incompressible case, a mapping with non-vanishing Jacobian does not always lead to a useful result: the region obtained may be simply-connected but multiply covered. This difficulty has not been experienced so far in some numerical examples which have been worked out. 
angles properly adjusted in relation to the free-stream Mach number. Indeed, it seems that after reaching the relation

$$
d z=\frac{d F}{w^{*}}-\frac{1}{4} \overline{w^{*} d F}
$$

the most natural development of the Kármán-l’sicn method is to leave $w^{*}(\zeta)$ quite free, as we have done here, instead of connecting it lefinitely with $w_{0}$, as was lonc by previous authors. The present method of leaving $k(\zeta)$ free seems to be the most general scheme.

If we deliberately want to introduce some singular points in $P$ by starting from a profile $P_{0}$ without a singular point, $|k(\zeta)|$ should be allowed to take the lim iting values specified in $(2.6)$; e.g., $k(\zeta)=0$ where $w_{0}(\zeta)=0$.

Although $k(\zeta)$ cannot be taken to be unity when the flow has a circulation, it should not depart from unity very much if the profiles $P$ and $P_{0}$ are not to differ much from each other. This is easily seen from a comparison of terms in (cf. (2.8))

$$
d z=k d \zeta-\frac{1}{4} \overline{\frac{w_{0}^{2}}{k} d \zeta .}
$$

They have the ratio (cf. (2.9) and (2.5))

$$
\lambda=\left(\begin{array}{c}
q \\
1+1 \frac{1+q^{2}}{1+}
\end{array}\right)^{2}=\frac{M^{2}}{\left(1+\sqrt{\left.1-M^{2}\right)^{2}}\right.},
$$

where $M$ is the local Mach number $q c$. This value is much smaller than unity, except for values of $M$ close to unity. ${ }^{\top}$ Hence. (4.4) is approximately the identity transformation if $k$ is very close to unity. This approximately preserves the shape of the profilc. during the transformation.

(b) Conformal mapping of compressible flows. If we make a conformal transformation of the $\zeta$-plane into the $\widetilde{\zeta}$-plane by the analytic relation

$$
\zeta=h(\tilde{\zeta})
$$

we are merely making a change of the auxiliary variable in (2.8)-(2.10). Indeed, we have

$$
\begin{aligned}
& x+i y=\int \tilde{k} d \tilde{\zeta}-\frac{1}{4} \int \frac{\overline{\tilde{w}_{0}^{2}(\tilde{\zeta})}}{\tilde{k}(\tilde{\zeta})} d \tilde{\zeta} \\
& \qquad \frac{2 q}{\sqrt{1+q^{2}}} e^{-i \theta}=\frac{\tilde{w}_{0}(\zeta)}{\tilde{k}(\tilde{\zeta})} \\
& \phi+i \psi=\tilde{F}(\tilde{\zeta})
\end{aligned}
$$

where

$$
\begin{aligned}
\tilde{F}(\tilde{\zeta}) & =F(\zeta) \\
\tilde{w}_{0}(\tilde{\zeta}) & =\tilde{F}^{\prime}(\tilde{\zeta})=F^{\prime}(\zeta) \frac{d \zeta}{d \tilde{\zeta}}
\end{aligned}
$$

\footnotetext{
7 Cf., Fig. 3 of Tsien's paper quoted in Footnote 3.
} 
and

$$
\tilde{k}(\tilde{\zeta})=k(\zeta) \frac{d \zeta}{d \tilde{\zeta}} .
$$

IVe note that the equations (4.7)-(4.9) are of the same nature as (2.8)-(2.10). However, the profile $\widetilde{P}_{0}$, into which the profiles $P$ and $P_{0}$ are mapped, may bear no resemblance at all to the original profiles. Indeed, there is no loss in generality in taking $P_{0}$ to be a circle. The relations (4.7)-(4.9) thus serve to transform the incompressible flow past a circle into a compressible flow past a profile of a quite arbitrary shape. Referring to (4.6) and (4.12) and to the fact that $k(\zeta)$ should be taken not far from unity, we see that $\widetilde{k}(\widetilde{\zeta})$ should be chosen so that it is not very much different from the derivative of the function mapping $P$ into a circle.

(c) Formulation of the direct problem. If we disregard the intermediate step of the $\zeta$-plane and drop the tilde, we have a mapping of the nature described in Section 2, but with $k(\zeta)$ so chosen that a circle will be mapped into some profile $P$. The function $k(\zeta)$ must satisfy the requirements established there, but it should not be very much different from the function $k_{0}(\zeta)$ which maps the circle into $P_{0}$ by the relation

$$
z=\int k_{0}(\zeta) d \zeta
$$

Thus for each profile $P$, the determination of the compressible flow past it is equivalent to the determination of a proper $k(\zeta)$ mapping it into a circle by (2.8), where $w_{0}(\zeta)$ is the flow past the curcle. There is no question about the existence of such a mapping function. It is clear that to each purely subsonic flow, where the approximation (2.4) is accurate enough and therefore $F=F\left(w^{*}\right)$, we may find a certain $k_{1}(\zeta)$ mapping the compressible flow into some incompressible flow. By considering successive conformal transformations, we can therefore always map the flow into a circle. The actual method of finding $k(\zeta)$ is all that remains to be done in the direct problem.

The theory of subsonic compressible flows (so long as the approximation (2.4) is valid) is now put on an equal footing with the incompressible flows. The inverse problem is complete, the direct problem of finding a mapping function for a given profile can only be solved (practically) by successive approximations, even in the incompressible case. ${ }^{8}$ To develop a method of successive approximations for the direct problem in the compressible case seems to be a natural next step.*

5. Application of von Mises' method of generating airfoils. In the incompressible case, von Mises transforms a circle in the $\zeta$-plane into an airfoil of a quite general shape by the transformation

$$
\frac{d z}{d \zeta}=\left(1-\frac{\lambda_{0}}{\zeta}\right)\left(1-\frac{\lambda_{1}}{\zeta}\right) \cdots\left(1-\frac{\lambda_{n}}{\zeta}\right)
$$

${ }^{8}$ Cf., e.g. the papers quoted in Footnote 5.

* Note added in proof: The essential difference of the two cases lies in the existence problem. While the existence of the incompressible flow follows from well-known results concerning the Laplace equation, very little seems to be known about the existence problem for compressible flows. 
where $\lambda_{1}, \cdots, \lambda_{r}$ are points inside the circle, and $\lambda_{0}$ is a point on the boundary (which transforms into the trailing edge of the airfoil). A similar method can be used here. In (2.8)-(2.10), we put

$$
k(\zeta)=\left(1-\frac{\lambda_{0}}{\zeta}\right)\left(1-\frac{\lambda_{1}}{\zeta}\right) \cdots\left(1-\frac{\lambda_{n}}{\zeta}\right)
$$

with the same general restrictions on the points $\lambda$. The condition that the points $\lambda$ are inside the circle is exactly the condition required of $k(\zeta)$ as stated in Section 2.

The condition (2.7) for the closure of the body has also its equivalence in the case considered by von Mises. There it is ${ }^{9}$

$$
\oint k(\zeta) d \zeta=0 .
$$

Here, it differs by the presence of another term. The condition that

$$
|k(\zeta)|>\left|\frac{1}{2} w_{0}(\zeta)\right|
$$

on the circle is the only additional restriction in the present case. As it is a mere inequality, there is no great difficulty in ensuring it to be satisfied.

The integration required in establishing the relation between $z$ and $\zeta$ can be readily performed, as it involves only rational functions. This ease of calculation, together with the flexibility of the choice of the points $\lambda$ in controlling the shape of the airfoil, are the advantages of the method of von Mises which are still preserved in the present application.

The author is greatly indebted to Dr. J. B. Diaz for very helpful discussions in the course of the investigation and to Professors W. Prager and K. O. Friedrichs for their interest and discussions.*

' Cf.'W. F. Durand, Aerodynamic theory, vol. 2, J. Springer, Berlin, 1933, p. 78, Eq. (20.4).

* Note added in proof: After the paper was completed and presented in a colloquium at Brown University, Professor E. Reissner informed the author that Professor A. Gelbart had recently presented a somewhat similar approach in a lecture at M.I.T. 